diminished by unlabelled agmatine, putrescine and ornithine. Similarly with DL- $\left[{ }^{14} \mathrm{C}\right]$ ornithine as the precursor labelling of homospermidine was decreased by unlabelled putrescine and arginine. From the results of these experiments it was considered that putrescine, for which multiple pathways exist, was the main intermediate in the synthesis of homospermidine.

In view of the symmetrical nature of the homospermidine molecule it was further considered that the other half of the molecule was also derived from putrescine. The biosynthesis may then involve a Schiff-base formation between putrescine and $\gamma$ aminobutyraldehyde, a metabolite of putrescine. Further reduction of the Schiff base would yield homospermidine. Such a mechanism is supported by the observed incorporation of ${ }^{3} \mathrm{H}$ from the medium into homospermidine, suggesting a nicotinamide nucleotide-dependent reduction. Also, in model systems containing $\gamma$-aminobutyraldehyde and putrescine, followed by reduction with $\mathrm{NaBH}_{4}$ or $\mathrm{Pt} / \mathrm{H}_{2}$, the formation of homospermidine was demonstrated. On the basis of these results a scheme for the biosynthesis of homospermidine in sandal is presented.

Kuttan, R. \& Radhakrishnan, A. N. (1970) Biochem. J. 117, 1015

Kuttan, R., Radhakrishnan, A. N., Spande, T. \& Witkop, B. (1971) Biochemistry 10, 361

Smith, T. A. (1970) Ann. N.Y. Acad. Sci. 171, 988

Tabor, H. \& Tabor, C. W. (1964) Pharmacol. Rev. 16, 245

\section{The Regulation of Nitrate Reductase in Neurospora crassa}

By K. N. Subramanian and G. J. Sorger (Department of Biology, McMaster University, Hamilton, Ont., Canada)

Nitrate is known to be necessary for the synthesis of nitrate reductase, and ammonium ion is known to inhibit the formation of this enzyme. The sites of action of nitrate and ammonium ions are unknown.

Neurospora mycelia were grown on ammonium ions as nitrogen source and subsequently induced by transfer to a medium containing nitrate and cycloheximide. Under these conditions nitrate reductase mRNA accumulated in the absence of protein synthesis and was measured by again transferring the mycelia to a medium containing nitrate and actinomycin $\mathrm{D}$ for $120 \mathrm{~min}$, to allow the formation of nitrate reductase by translation of the existing mRNA. Nitrate was essential for the accumulation of nitrate reductase mRNA, and ammonium ions prevented this accumulation. Ammonium ions had no effect on the translation of the accumulated nitrate reductase mRNA. Ammonium ion is obviously acting as corepressor and nitrate as co-inducer.
Mycelia in which nitrate reductase had been maximally induced by nitrate were transferred to fresh media containing different nitrogen sources. Nitrate stabilized the enzyme in vivo, as shown by contrast with the rapid loss of enzyme activity on transfer of mycelia to a medium containing no nitrogen source. Ammonium ions decreased the stability of nitrate reductase in vivo. Nitrate did not antagonize this effect of ammonium ions, which suggests that nitrate and ammonia might act at two different sites. Cycloheximide partially protected nitrate reductase from loss of activity after the removal of nitrate or the addition of ammonium ions.

In contrast with the tobacco cell-culture system of Heimer \& Filner (1971) we have found no evidence for inhibition of nitrate uptake by ammonium ions in Neurospora.

Heimer, Y. M. \& Filner, P. (1971) Biochim. Biophys. Acta 230, 362

Role of Light in the Induction of Nitrate Reductase and Nitrite Reductase in Rice Seedlings

By S. K. SAwhney and M. S. NAIK (Division of Biochemistry, Indian Agricultural Research Institute, New Delhi-12, India)

Assimilatory nitrate reductase is a substrateinduced enzyme both in higher plants and microorganisms, but in green tissues light is also essential for the synthesis of the enzyme (Travis et al., 1970a). The precise role of light is obscure at present (Beevers \& Hageman, 1969; Travis et al., 1970b).

In germinating rice (Oryzae sativa, var. $\mathrm{TN}-1$ ) seeds light was not found to be necessary for the synthesis of nitrate reductase and nitrite reductase up to 7 days. After this period light substantially stimulated the synthesis of the former enzyme but was without effect on the latter. Subsequent transfer of seedlings to the dark resulted in a continuous decline in the activity of nitrate reductase. Lower activity in the absence of light could not be attributed to the production of inhibitors or to a depressed uptake of the inducer. NADH oxidase activity was higher in dark-grown seedlings, but this also did not account for the lower NADH-nitrate reductase activity.

Beevers, L. \& Hageman, R. H. (1969) Annu. Rev. Plant Physiol. 20, 495

Travis, R. L., Jordan, W. R. \& Huffaker, R. C. (1970a) Physiol. Plant. 23, 678

Travis, R. L., Huffaker, R. C. \& Key, J. L. (1970b) Plant Physiol. 46, 800 